PHYSICAL REVIEW D 80, 083508 (2009)

\title{
Zipping and unzipping of cosmic string loops in collision
}

\author{
H. Firouzjahi* \\ School of Physics, Institute for Research in Fundamental Sciences (IPM), P.O. Box 19395-5531, Tehran, Iran \\ J. Karouby ${ }^{\dagger}$ \\ Physics Department, McGill University, Montreal, H3A 2T8, Canada
}

S. Khosravi ${ }^{\ddagger}$

Physics Department, Faculty of Science, Tarbiat Mo'alem University, Tehran, Iran

School of Astronomy, Institute for Research in Fundamental Sciences (IPM), Tehran, Iran

R. Brandenberger ${ }^{\S}$

Physics Department, McGill University, Montreal, H3A 2T8, Canada

Theory Division, CERN, CH-1211 Genève, Switzerland

(Received 7 August 2009; published 7 October 2009)

\begin{abstract}
In this paper the collision of two cosmic string loops is studied. After collision junctions are formed and the loops are entangled. We show that after their formation the junctions start to unzip and the loops disentangle. This analysis provides a theoretical understanding of the unzipping effect observed in numerical simulations of a network of cosmic strings with more than one type of cosmic strings. The unzipping phenomena have important effects in the evolution of cosmic string networks when junctions are formed upon collision, such as in a network of cosmic superstrings.
\end{abstract}

DOI: 10.1103/PhysRevD.80.083508

PACS numbers: $98.80 .-\mathrm{k}, 98.80 . \mathrm{Cq}$

\section{INTRODUCTION}

Cosmic strings are copiously produced at the end of brane inflation $[1,2]$ (for reviews see e.g. [3-6]). These strings are in the form of fundamental strings (F strings), D1-branes (D strings) or their bound states. F and D strings can combine to form bound states- $(p, q)$ strings-which are constructed from $p \mathrm{~F}$ strings and $q \mathrm{D}$ strings on top of each other. Because of charge conservation, when two $(p, q)$ cosmic strings intersect generally a junction is formed. This is in contrast to what happens in the case of $\mathrm{U}(1)$ gauge cosmic strings: When two $\mathrm{U}(1)$ gauge cosmic strings intersect, they usually exchange partners and intercommute with probability close to unity. In this view, the formation of junctions may be considered as a novel feature of the network of cosmic superstrings. Networks of strings with junctions have interesting physical properties, such as the formation of multiple images $[7,8]$ and nontrivial gravity wave emission $[9,10]$. Different theoretical aspects of $(p, q)$ string construction were studied in [11$16]$ while the cosmological evolution of a string network with junctions has been investigated in [17].

The evolution of a network containing two types of cosmic strings was studied by Urrestilla and Vilenkin [18]. In their model, the cosmic strings are two types of $\mathrm{U}(1)$ gauge strings with interactions between them. Let us label these strings as A and B strings. Because of the

\footnotetext{
*firouz@ipm.ir

${ }^{\dagger}$ karoubyj@physics.mcgill.ca

*khosravi@ipm.ir

§hb@physics.mcgill.ca
}

interaction, the strings cannot exchange partners and a bound state, string $\mathrm{AB}$, will form if the strings are not moving too fast. It was shown that the length and the distribution of the string network are dominated by the original A and B strings and there is a negligible contribution to the string network length and population from the bound states strings $\mathrm{AB}$. This can be understood based on the following two reasons. First, the junctions may not form if the colliding strings are moving very fast so they can simply pass through each other [19-24]. Second and more curiously, if the junctions are formed, they start to unzip during the evolution. The process of zipping and unzipping of cosmic strings in collision is a nontrivial dynamical property. Our aim here is to provide some theoretical understanding of how this process happens in the collision of cosmic strings loops.

\section{THE SETUP}

Here we provide our setup. We consider two cosmic string loops moving in opposite directions. At the time of the collision, junctions are formed. This can be viewed as a generalization of straight strings collision [20-22]. However, due to topological constraints, there are new nontrivial effects which can lead to the unzipping of junctions. This is unlike what happens in the case of straight strings, where for two colliding straight strings, once the junctions are formed, they will always grow with time and do not unzip [20-22].

In order to simplify the analysis, we assume the colliding loops have equal tensions and radii and that the planes 
they span are parallel. Choosing the center of mass frame, we take the loops to be in the $x-y$ plane and assume they are moving along the $z$ axis with speed $\pm v$. A schematic view of the collision is shown in Fig. 1. The collision happens at $t=0, z=0$. There are two collision points. The angle of collision $2 \alpha$ is defined as the angle between the tangential lines to the loops at the points of collision.

We choose the incoming strings to be of the form of simple loops in their rest frames:

$$
R(t)=R_{0} \cos \left(\frac{t-t_{0}}{R_{0}}\right)
$$

This may not be a realistic configuration, but due to the complexity of the collision analysis, this ansatz is illustrative enough to capture the unzipping effect. Here $t_{0}$ is the phase at the time of collision and the radius at collision time is $R_{0} \cos \left(t_{0} / R_{0}\right)$.

After the collision, four junctions are formed (in Fig. 1 they are denoted by $A, B, C$ and $D$ ). Because of symmetry, one expects that junctions $A$ and $B$ and junctions $C$ and $D$ evolve similarly but in opposite directions. On each junction, there are three string legs; two of them are the incoming strings and the third is the newly formed string with tension $\mu_{3}$. As explained above, we assume that the incoming loops have equal tensions: $\mu_{1}=\mu_{2}$. Later we will see that for a junction to form one requires that $2 \mu_{1}>$ $\gamma \mu_{3}$.

Because of the symmetry, the third string is stationary and is oriented either along the $y$ axis ( $y$ link) or the $x$ axis ( $x$ link). The orientation of the third string is controlled by the angle $\alpha$. For small $\alpha$ (roughly $0<\alpha<\pi / 4$ ) we expect a $y$-link junction and for a larger value of $\alpha$ an $x$-link junction. For the discussion below and in Fig. 1 we consider a $y$-link junction.
Guided by the causality and the symmetry of the problem, one expects that, after collision, the entangled loops are divided into two secondary loops, the external loop and the internal loop. The external and the internal loops are connected by the newly formed strings with tension $\mu_{3}$. Given the symmetry of the setup, a nice feature of the internal and external loops is that half of each is from the first string and the other half is from the second string. There are four kinks on each secondary loop separating the newly formed arcs from the parts of the old loops which do not yet feel the presence of the junctions (by causality). As we shall see, these nontrivial topological constraints between the internal and external loops play an essential role in the unzipping process.

At the time of collision the system has a nonzero angular momentum around the axis of collision. However, we do not expect the angular momentum to play an important role in the unzipping process. As we shall see below, the unzipping process is determined by forces in the place of the strings, whereas angular momentum induces forces in the orthogonal direction. In addition, these forces will vanish at the local of the junctions.

The world sheet of each string is described by a temporal coordinate $\tau$ and a string length parameter $\sigma_{i}$. We take each string to have its own $\sigma_{i}$ parameter. Our convention for the orientation of $\sigma_{i}$ is that on a given loop, whether an original colliding loop or a secondary loop, the $\sigma_{i}$ coordinate increases counterclockwise from 0 to $2 \pi$. For example, at the time of collision, the point $M$ in Fig. 1 has $\sigma_{1}=\gamma R_{0} \alpha$ on string 1 and $\sigma_{2}=\gamma R_{0}(\pi-\alpha)$ on string 2 . Similarly, the point $N$ has $\sigma_{1}=\gamma R_{0}(2 \pi-\alpha)$ on string 1 and $\sigma_{2}=\gamma R_{0}(\pi+\alpha)$ on string 2. Here $\gamma$ is the Lorentz factor, $\gamma^{-2}=1-v^{2}$, which shows up due to the boost from the string rest frame to the center of mass frame.

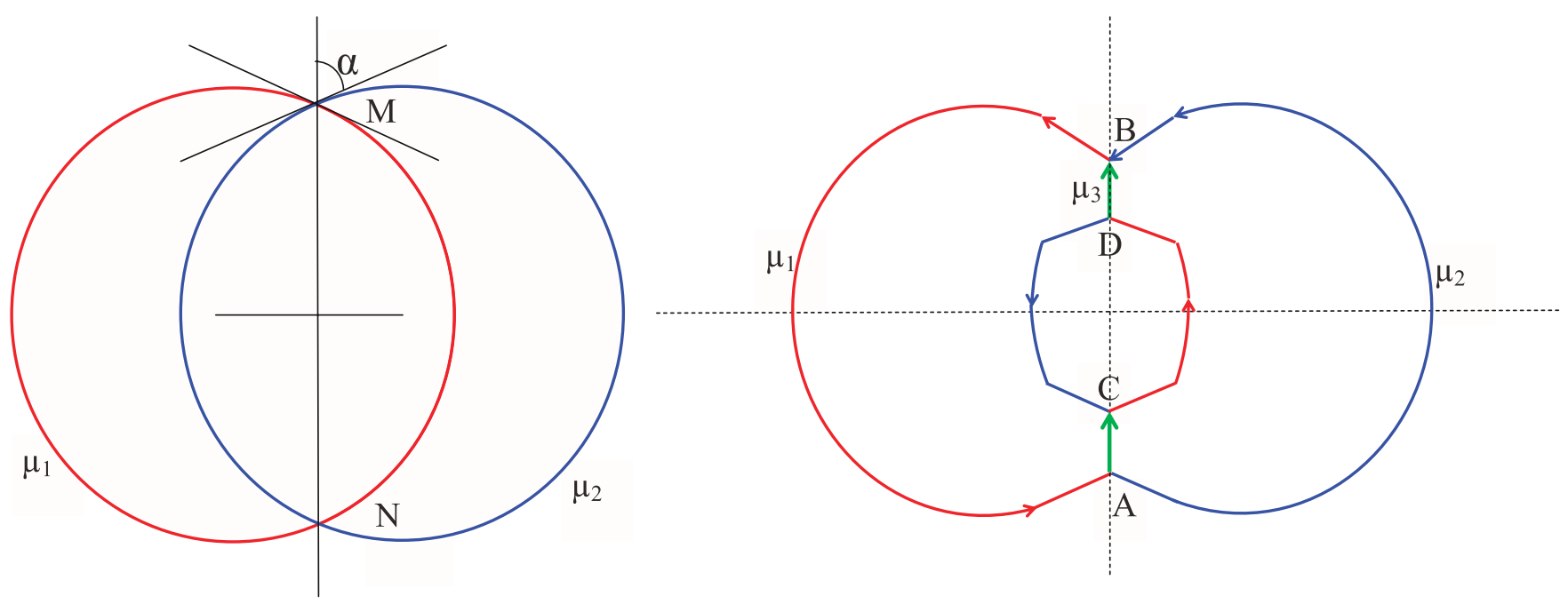

FIG. 1 (color online). A schematic view of the loops at the time of collision (left) and after collision (right). The arrows in the right figure indicate the directions in which the $\sigma_{i}$ coordinate increases. We use the convention that on a loop $\sigma_{i}$ runs counterclockwise. There are four junctions and eight kinks in total. 
Finally on the third string, $\sigma_{3}$ increases from south to north.

One complexity of dealing with loops in collision is the orientation of the $\sigma_{i}$ coordinate at junctions. We follow the prescription of [25] and use the sign parametrization for $\delta_{i}$ according to which $\delta_{i}$ can take values \pm 1 . If the value of $\sigma_{i}$ of a particular string increases (decreases) towards the junction, we assign $\delta_{i}=+1\left(\delta_{i}=-1\right)$. With this prescription, the two ends of a piece of string ending in two neighboring junctions have opposite $\delta$ parameters. The arrows in Fig. 1 indicate this prescription. Since it is important for the later analysis, we now give the values of $\delta_{i}$ at each junction:

$$
\begin{aligned}
& A:\left|\begin{array}{l|l}
\delta_{1}=+1 \\
\delta_{2}=-1, \\
\delta_{3}=-1
\end{array} \quad B:\right| \begin{array}{l}
\delta_{1}=-1 \\
\delta_{2}=+1, \\
\delta_{3}=+1
\end{array}, \\
& C: \quad \begin{array}{l|l}
\delta_{1}=-1 \\
\delta_{2}=+1, \\
\delta_{3}=+1
\end{array} \quad D: \quad \begin{array}{l}
\delta_{1}=+1 \\
\delta_{2}=-1 \\
\delta_{3}=-1
\end{array}
\end{aligned}
$$

We consider a flat space-time background. The induced metric $\gamma_{i a b}$ on each string is given by

$$
\gamma_{i a b}=\eta_{\mu \nu} \partial_{a} x_{i}^{\mu} \partial_{b} x_{i}^{\nu} .
$$

Here and in the following, we reserve $\{a, b\}=\left\{\tau, \sigma_{i}\right\}$ for the string world-sheet indices while Greek indices represent the four-dimensional space-time coordinates. Furthermore, $x_{i}^{\mu}$ stands for the position of the $i$ th string in the target space-time.

After collision, the junction points correspond to the intersection of three segments of strings: two from the colliding loops and one from the bound state string that appears after collision. Including the $\delta$ parametrization on each segment of strings, the Nambo-Goto action describing the dynamics of the strings positions $x_{i}^{\mu}$ and the evolution of junction points is [25]

$$
\begin{aligned}
S= & -\sum_{i} \mu_{i} \delta_{i} \int d \tau \int d \sigma_{i} \sqrt{-x_{i}^{\prime 2} \dot{x}_{i}^{2}} \theta\left(s_{i}^{B_{i}}(\tau)-\sigma_{i}\right) \\
& \times \theta\left(-s_{i}^{A_{i}}(\tau)+\sigma_{i}\right)+\sum_{i} \sum_{J} \int d \tau f_{i \mu}^{J} . \\
& \times\left[x_{i}^{\mu}\left(\tau, s_{i}^{J}(\tau)\right)-X_{J}^{\mu}(\tau)\right] .
\end{aligned}
$$

Here an overdot and a prime denote derivatives with respect to $\tau$ and $\sigma$, respectively. The function $s_{i}^{J}(\tau)$ indicates the value of the $\sigma_{i}$ coordinate for the $i$ th string at the junction $J$. The theta functions indicate the fact that each piece of string exists only between junctions $B_{i}$ and $A_{i}$. In this notation, the $\sigma_{i}$ coordinate for the piece of the string which stretches from junctions $B_{i}$ to $A_{i}$ is increasing from $B_{i}$ to $A_{i}$ and

$$
s_{i}^{B_{i}}(\tau) \leq \sigma_{i} \leq s_{i}^{A_{i}}(\tau) .
$$

It should be noted that in our case $\left\{A_{i}, B_{i}\right\}$ collectively stand for the junction points $J$ with

$$
J \in\{A, B, C, D\}
$$

in Fig. 1. Finally, the functions $f_{i \mu}^{J}$ are the Lagrange multipliers which enforce the constraints that at the junction $J$, the three strings meet and

$$
x_{i}\left(s_{i}^{J}(\tau), \tau\right)=X^{J}(\tau),
$$

where $X^{J}(\tau)$ is the junction position in the target spacetime.

As explained above, the value of the $\sigma_{i}$ coordinate for the $i$ th string at junction $J$ is given by the function $s_{i}^{J}(\tau)$. It is a dynamical variable which controls the evolution of the junction. For example, at junction $B$ in Fig. 1 the process of zipping for the string $\mu_{3}$ happens when $\dot{s}_{3}^{B}(\tau)>0$ whereas its unzipping happens when $\dot{s}_{3}^{B}(\tau)$ vanishes at some time during evolution and $\dot{s}_{3}^{B}(\tau)<0$ afterwards. Our goal in the next section is to find the dynamical equations for $\dot{s}_{i}^{J}$ to understand the process of zipping and unzipping of strings in junctions.

The derivation of the equations of motion coming from action (4) is given in [25]. Here we summarize the equations which are necessary for our colliding loop analysis.

We impose the conformal temporal gauge on the string world sheet, namely, $X_{i}^{0}=t=\tau$ and $\gamma_{i 0 \sigma}=0$. This is equivalent to

$$
\dot{\mathbf{x}}_{i} \cdot \mathbf{x}_{i}^{\prime}=0, \quad \dot{\mathbf{x}}_{i}^{2}+\mathbf{x}_{i}^{\prime 2}=1,
$$

where the $\mathbf{x}_{i}$ represent the spatial components of the $i$ th string. The solution of string equation of motion $\ddot{\mathbf{x}}_{i}-\mathbf{x}_{i}^{\prime \prime}=$ 0 , as usual, is given in terms of left- and right-mover waves:

$$
\mathbf{x}_{i}(t, \sigma)=\frac{1}{2} \mathbf{a}_{i}\left(\frac{\sigma+t}{2}\right)+\frac{1}{2} \mathbf{b}_{i}\left(\frac{\sigma-t}{2}\right)
$$

with $\mathbf{a}_{i}^{\prime 2}=\mathbf{b}_{i}^{\prime 2}=1$. Imposing the junction conditions obtained from varying the action (4), one can find expressions for $\mathbf{a}_{i}^{\prime}$ and $\mathbf{b}_{i}^{\prime}$ at the position of the junction $J$. Imposing the conditions $\mathbf{a}_{i}^{\prime 2}=\mathbf{b}_{i}^{\prime 2}=1$ one finds the following equations for $s_{i}^{J}$ :

$$
\dot{s}_{i}{ }^{J}=\delta_{i}\left(1-\frac{\mu M_{i}\left(1-c_{i}^{J}\right)}{\mu_{i} \sum_{k} M_{k}\left(1-c_{k}\right)}\right),
$$

where $\mu \equiv \sum_{i} \mu_{i} \quad$, and

$$
M_{i}=\mu_{1}^{2}-\left(\mu_{j}-\mu_{k}\right)^{2}, \quad c_{i}^{J}(t)=Y_{i} . Y_{k}
$$

with $i \neq j \neq k$ and

$$
Y_{j}= \begin{cases}\mathbf{b}_{j}^{\prime} & \text { if } \delta_{i}=+1, \\ -\mathbf{a}_{j}^{\prime} & \text { if } \delta_{i}=-1 .\end{cases}
$$

It should be noted that the $Y_{i}$ are constructed at the point of each junction $J$ where $\sigma_{i}=s_{i}^{J}(\tau)$. 
Finally, energy conservation at each junction $J=$ $\{A, B, C, . D\}$ requires that

$$
\delta_{1}^{J} \mu_{1} \dot{s}_{1}^{J}+\delta_{2}^{J} \mu_{2} \dot{s}_{2}^{J}+\delta_{3}^{J} \mu_{3} \dot{s}_{3}^{J}=0 .
$$

One can check that this also follows from Eq. (10).

\section{ZIPPING AND UNZIPPING}

Here we study in detail the equations of motion for $s_{i}(t)$. Let us start with junction B. At the time of collision $s_{3}^{B}(0)=0$. For the junction to form, $s_{3}^{B}(t)$ should be increasing initially. For unzipping to happen, $s_{3}^{B}(t)$ should come to a stop (i.e. $\dot{s}_{3}^{B}=0$ ) at some time $t=t_{u}^{B}$ corresponding to the time of unzipping at junction B. Then $s_{3}^{B}(t)$ decreases. Similarly, $s_{3}^{D}(0)=0$ initially and after collision $s_{3}^{D}(t)$ decreases, reaching a minimum negative value before turning back. The unzipping at junction $\mathrm{D}$ happens at $t=$ $t_{u}^{D}$ (when $\dot{s}_{3}^{D}=0$ ). Interestingly, we find that $t_{u}^{D} \neq t_{u}^{B}$. The loops disentangle and separate from each other at the time $t=t_{f}$ when the junctions B and D meet, corresponding to $s_{3}^{D}\left(t_{f}\right)=s_{3}^{B}\left(t_{f}\right)$. As we shall see, the loop disentanglement does not happen when $s_{3}^{D}(t)=s_{3}^{B}(t)=0$. It turns out that $s_{3}^{D}\left(t_{f}\right)=s_{3}^{B}\left(t_{f}\right)<0$. Because of our symmetric construction, the same arguments go through for junctions $\mathrm{A}$ and $\mathrm{C}$ and we can restrict the analysis to the pair of junctions B and $\mathrm{D}$.

Going to the center of mass frame, it follows from Eq. (1) that

$$
\mathbf{x}_{1,2}=\left(\begin{array}{c}
\mp b+R_{0} \cos \left(\frac{t-t_{0}}{\gamma R_{0}}\right) \cos \left(\frac{\sigma_{1,2}}{\gamma R_{0}}\right) \\
R_{0} \cos \left(\frac{t-t_{0}}{\gamma R_{0}}\right) \sin \left(\frac{\sigma_{1,2}}{\gamma R_{0}}\right) \\
\pm v t
\end{array}\right),
$$

where the impact parameter $2 b$ is the separation between the centers of the loops (see Fig. 1). Decomposing $\mathbf{x}_{i}$ into left movers as in Eq. (9) yields

$$
\begin{array}{r}
\mathbf{a}_{1,2}^{\prime}=\left(\begin{array}{c}
-\gamma^{-1} \sin \left(\frac{\sigma_{1,2}+t-t_{0}}{\gamma R_{0}}\right) \\
\gamma^{-1} \cos \left(\frac{\sigma_{1,2}+t-t_{0}}{\gamma R_{0}}\right) \\
\pm v
\end{array}\right), \\
\mathbf{b}_{1,2}^{\prime}=\left(\begin{array}{c}
-\gamma^{-1} \sin \left(\frac{\sigma_{1,2}-t+t_{0}}{\gamma R_{0}}\right) \\
\gamma^{-1} \cos \left(\frac{\sigma_{1,2}-t+t_{0}}{\gamma R_{0}}\right) \\
\bar{\mp} \boldsymbol{v}
\end{array}\right) .
\end{array}
$$

For the third string which stretches between the $\mathrm{D}$ and $\mathrm{B}$ junctions one has (following the arrows in Fig. 1 where $\sigma_{3}$ increases from south to north)

$$
\mathbf{x}_{3}=\left(0, \sigma_{3}, 0\right), \quad \mathbf{a}_{3}^{\prime}=\mathbf{b}_{3}^{\prime}=(0,1,0) .
$$

Let us start again with the junction B. Based on symmetry considerations (both loops have equal tensions and radii) one expects that $\dot{s}_{1}^{B}(t)=-\dot{s}_{2}^{B}(t)$. From Eq. (10) one can check that $\dot{s}_{1}^{B}(t)=-\dot{s}_{2}^{B}(t)$ is a consistent solution. This in turn leads to $s_{1}^{B}(t)+s_{2}^{B}(t)=s_{1}^{B}(0)+s_{2}^{B}(0)$. However, at the time of collision, $s_{1}^{B}(0)=\gamma R_{0} \alpha$ and $s_{2}^{B}(0)=\gamma R_{0}(\pi-$ $\alpha)$ so

$$
s_{1}^{B}(t)=-s_{2}^{B}(t)+\gamma R_{0} \pi .
$$

Using the energy conservation equation (13) one obtains

$$
s_{3}^{B}(t)=-\frac{2 \mu_{1}}{\mu_{3}}\left[s_{2}^{B}(t)-\gamma R_{0}(\pi-\alpha)\right] .
$$

The dynamical process of zipping, unzipping and loop disentanglement is controlled by the functions $s_{3}^{B}(t)$ and $s_{3}^{D}(t)$. To obtain the differential equation for $\dot{s}_{3}^{B}$, we first need to calculate the quantities $c_{i}(t)$ at junction $\mathrm{B}$. One has

$$
c_{1}=\mathbf{b}_{2}^{\prime} \cdot \mathbf{b}_{3}^{\prime}=\gamma^{-1} \cos \left(\frac{s_{2}^{B}(t)-t+t_{0}}{\gamma R_{0}}\right)
$$

and

$$
c_{2}=-\mathbf{a}_{1}^{\prime} \cdot \mathbf{b}_{3}^{\prime}=\gamma^{-1} \cos \left(\frac{s_{1}^{B}(t)+t-t_{0}}{\gamma R_{0}}\right)=c_{1},
$$

where to obtain the final relation, use was made of Eq. (17). Similarly, one obtains

$$
c_{3}^{B}=-\mathbf{a}_{1}^{\prime} \cdot \mathbf{b}_{2}^{\prime}=-1+2 \gamma^{-2} \cos ^{2}\left(\frac{s_{2}^{B}(t)-t+t_{0}}{\gamma R_{0}}\right) .
$$

With these values of $c_{i}(t)$ and using Eq. (10), one obtains

$$
\dot{s}_{3}^{B}=\frac{2 \mu_{1} \gamma^{-1} \cos \left(\frac{\mu_{3} s_{3}^{B}(t)}{2 \mu_{1} \gamma R_{0}}+\alpha+\frac{t-t_{0}}{\gamma R_{0}}\right)-\mu_{3}}{2 \mu_{1}-\mu_{3} \gamma^{-1} \cos \left(\frac{\mu_{3} s_{3}^{B}(t)}{2 \mu_{1} \gamma R_{0}}+\alpha+\frac{t-t_{0}}{\gamma R_{0}}\right)},
$$

where to get the final answer, the relation (18) has been used to eliminate $s_{2}^{B}(t)$ in favor of $s_{3}^{B}(t)$.

To check the validity of the above expression, one can show that in the limit where $R_{0} \rightarrow \infty$, it reduces to the result of [20] for collision of two infinite straight strings.

Following the same steps, for the junction D one finds

$$
\dot{s}_{3}^{D}=-\frac{2 \mu_{1} \gamma^{-1} \cos \left(\frac{\mu_{3} s_{3}^{D}(t)}{2 \mu_{1} \gamma R_{0}}+\alpha-\frac{t-t_{0}}{\gamma R_{0}}\right)-\mu_{3}}{2 \mu_{1}-\mu_{3} \gamma^{-1} \cos \left(\frac{\mu_{3} s_{3}^{D}(t)}{2 \mu_{1} \gamma R_{0}}+\alpha-\frac{t-t_{0}}{\gamma R_{0}}\right)} .
$$

Comparing the equations for $\dot{s}_{3}^{B}$ and $\dot{s}_{3}^{D}$, we observe that $s_{3}^{B} \rightarrow-s_{3}^{D}$ under time reversal $t-t_{0} \rightarrow-\left(t-t_{0}\right)$.

One can check that for the junctions A and B the evolution of $\dot{s}_{3}^{A}$ and $\dot{s}_{3}^{C}$ is identical to that of $\dot{s}_{3}^{B}$ and $\dot{s}_{3}^{D}$, with a sign difference as expected due to our symmetric construction.

With some effort, one can solve Eqs. (22) and (23) with the answer

$$
\begin{gathered}
\frac{s_{3}^{B}}{R_{0}}-\sin \left(\frac{\mu_{3} s_{3}^{B}}{2 \mu_{1} \gamma R_{0}}+\alpha+\frac{t-t_{0}}{\gamma R_{0}}\right) \\
=-\sin \left(\alpha-\frac{t_{0}}{\gamma R_{0}}\right)-\frac{\mu_{3}}{2 \mu_{1} R_{0}} t
\end{gathered}
$$

which expresses $s_{3}^{B}(t)$ implicitly as a function of $t$. A similar equation holds for $s_{3}^{D}$ with $\left(t, t_{0}\right) \rightarrow-\left(t, t_{0}\right)$. 
The above implicit equations for $s_{3}^{B}$ and $s_{3}^{D}$ cannot be solved explicitly to obtain the variables as functions of $t$. However, some insight can be obtained by looking at the form of Eq. (22). For the junction B to materialize at $t=0$, we need that $\dot{s}_{3}^{B}(0)>0$. This requires that $\gamma \mu_{3}<2 \mu_{1}$ and

$$
\left(\alpha-\frac{t_{0}}{\gamma R_{0}}\right)<\alpha_{c} \equiv \cos ^{-1}\left(\frac{\mu_{3} \gamma}{2 \mu_{1}}\right) .
$$

Interestingly, this is the same junction formation condition as for the collision of straight strings [20] where $R_{0} \rightarrow \infty$. This is understandable, since the collision and junction formation is a local effect and at the points of collision large loops may be approximated as straight strings. On the other hand, for the junction D to materialize after collision, one expects that $\dot{s}_{3}^{D}(0)<0$ which yields

$$
\left(\alpha+\frac{t_{0}}{\gamma R_{0}}\right)<\alpha_{c} .
$$

Interestingly, when $t_{0} \neq 0$, Eq. (26) is stronger a condition than Eq. (25).

Once the junction B is formed, it grows until the time $t_{u}^{B}$ of unzipping, when the argument inside the cos function in Eq. (22) becomes equal to $\alpha_{c}$ and $\dot{s}_{3}^{B}=0$. As time goes by, the argument inside the cos function increases, $\dot{s}_{3}^{B}$ becomes negative and the junction $B$ turns back. A similar argument applies to junction $\mathrm{D}$ except that the unzipping happens at the time $t=t_{u}^{D}$, and due to the time asymmetry in Eqs. (22) and (23), $t_{u}^{D} \neq t_{u}^{B}$. Below we will demonstrate that $t_{u}^{D}>t_{u}^{B}$. After $t>t_{u}^{D}$, the junctions $\mathrm{B}$ and $\mathrm{D}$ move towards each other. The loops disentangle at the time $t=t_{f}$ when the junctions meet, corresponding to $s_{3}^{B}\left(t_{f}\right)=s_{3}^{D}\left(t_{f}\right)$. In Fig. 2 we have plotted the shapes of $s_{3}^{B}$ and $s_{3}^{D}$ for some given parameter values of $\alpha, \gamma, \mu_{1}, \mu_{2}$ and $R_{0}$. The left figure indicates that $s_{3}^{B}\left(s_{3}^{D}\right)$ increases (decreases) initially and then come to a halt, indicating the time of unzipping.

Here we would like to find the time of unzipping and loop disentanglement. Consider junction B. At the time
$t=t_{u}^{B}$ of unzipping one obtains from $\dot{s}_{3}^{B}=0$ that

$$
\frac{s_{3}^{B}\left(t_{u}^{B}\right)}{R_{0}}=\frac{2 \mu_{1} \gamma}{\mu_{3}}\left(\alpha_{c}-\alpha-\frac{t_{u}^{B}-t_{0}}{\gamma R_{0}}\right) .
$$

Plugging this into Eq. (24) gives the unzipping time

$$
\begin{aligned}
\frac{t_{u}^{B}}{R_{0}}= & \left(1-\frac{\mu_{3}^{2}}{4 \mu_{1}^{2}}\right)^{-1}\left\{\gamma\left(\alpha_{c}-\alpha\right)+\frac{t_{0}}{R_{0}}\right. \\
& \left.+\frac{\mu_{3}}{2 \mu_{1}}\left[\sin \left(\alpha-\frac{t_{0}}{\gamma R_{0}}\right)-\sqrt{1-\frac{\gamma^{2} \mu_{3}^{2}}{4 \mu_{1}^{2}}}\right]\right\} .
\end{aligned}
$$

To find the unzipping time for junction $\mathrm{D}$, we note that after junction formation the argument inside the cos function in Eq. (23) decreases with time. It becomes negative and the unzipping for junction $\mathrm{D}$ happens when the expression inside the cos function becomes equal to $-\alpha_{c}$. With this consideration and following the steps as above yields

$$
\begin{aligned}
\frac{t_{u}^{D}}{R_{0}}= & \left(1-\frac{\mu_{3}^{2}}{4 \mu_{1}^{2}}\right)^{-1}\left\{\gamma\left(\alpha_{c}+\alpha\right)+\frac{t_{0}}{R_{0}}\right. \\
& \left.-\frac{\mu_{3}}{2 \mu_{1}}\left[\sin \left(\alpha+\frac{t_{0}}{\gamma R_{0}}\right)+\sqrt{1-\frac{\gamma^{2} \mu_{3}^{2}}{4 \mu_{1}^{2}}}\right]\right\} .
\end{aligned}
$$

Equations (28) and (29) are implicit equations which relate $t_{u}^{B}$ and $t_{u}^{D}$ to the tensions $\mu_{i}$, the incoming angle of collision $\alpha$, the velocity $\gamma$ and the initial loop phase $t_{0}$. It is not easy to see how $t_{u}^{B}$ and $t_{u}^{D}$ vary as one varies these parameters simultaneously. As a simple treatment, let us take $\mu_{i}$ and $\gamma$ as fixed properties of a network of cosmic strings and consider the unzipping times as functions of $\alpha$ and $t_{0}$ (which may be considered as random parameters for the network evolution). If one increases $t_{0}>0$ while keeping $\alpha$ fixed, then both $t_{u}^{B}$ and $t_{u}^{D}$ increase. There is a limit on how large $t_{0}$ can be. This is determined by Eq. (26). The dependence of the unzipping on $\alpha$ is more nontrivial. From Eqs. (28) and (29) we note that the dependence of these

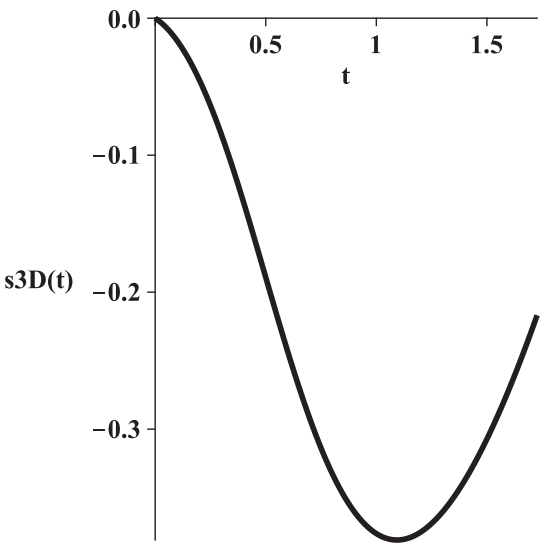

FIG. 2. Here the numerical solutions for $s_{3}^{B}(t)$ (left) and $s_{3}^{D}$ (right) are plotted for $\gamma=1.1, \mu_{1} / \mu_{3}=0.7, \alpha=\pi / 8$ and $t_{0}=0.1$ in units where $R_{0}=1$. The unzipping for junction B (D) happens when $s_{3}^{B}\left(s_{3}^{D}\right)$ reaches a maximum (minimum) value. In this example, the loop disentanglement happens at $t_{f} \simeq 1.4$ before the loops shrink at $t_{\text {shrink }} \simeq 1.7$. 
times on $\alpha$ is not symmetric. For a fixed value of $t_{0}$, then as $\alpha$ increases, $t_{u}^{D}$ increases while $t_{u}^{B}$ decreases almost linearly with $\alpha$. Again, there is a limit on how big $\alpha$ can be, which is determined by Eq. (26).

It is instructive to see which of the junctions B or D starts to unzip sooner. From the above two equations, the difference in the unzipping times is calculated to be

$$
\begin{aligned}
\frac{t_{u}^{D}-t_{u}^{B}}{R_{0}}= & 2 \gamma \alpha\left(1-\frac{\mu_{3}^{2}}{4 \mu_{1}^{2}}\right)^{-1} \\
& \times\left[1-\frac{\mu_{3}}{2 \mu_{1} \gamma} \cos \left(\frac{t_{0}}{\gamma R_{0}}\right) \frac{\sin \alpha}{\alpha}\right] .
\end{aligned}
$$

Since $\sin \alpha / \alpha$ is always less than unity we see that $t_{u}^{D}>t_{u}^{B}$. This means that the junction $B$ which holds the external large arcs unzips sooner than the junction $\mathrm{D}$ which holds the internal small arcs. Keeping all other parameters fixed, by increasing the angle of collision $\alpha$, the difference in unzipping times increases almost linearly with $\alpha$.

The time $t_{f}$ of loop disentanglement is given by $s_{3}^{B}\left(t_{f}\right)=$ $s_{3}^{D}\left(t_{f}\right)$. Using Eq. (24) and the similar equation for $s_{3}^{D}$ gives

$$
\begin{aligned}
\frac{2 \gamma \mu_{1}}{\mu_{3}} \cos ^{-1} \Gamma- & \cos \left(\frac{t-t_{0}}{\gamma R_{0}}\right) \sqrt{1-\Gamma^{2}}-\frac{2 \gamma \mu_{1} \alpha}{\mu_{3}} \\
+ & \sin \alpha \cos \left(\frac{t_{0}}{\gamma R_{0}}\right)=0,
\end{aligned}
$$

where

$$
\Gamma \equiv\left[\frac{\mu_{3} t /\left(2 \mu_{1} R_{0}\right)-\cos \alpha \sin \left(\frac{t_{0}}{\gamma R_{0}}\right)}{\sin \left(\frac{t-t_{0}}{\gamma R_{0}}\right)}\right] .
$$

This is an implicit equation for $t_{f}$ which should be solved in terms of $\mu_{i}, \gamma, \alpha, t_{0}$ and $R_{0}$. For this to make sense, we demand that $t_{f}-t_{0}<\pi R_{0} / 2$ before the loops shrink to zero.

\section{DISCUSSION}

In this paper we have provided a theoretical understanding of the zipping-unzipping phenomena in cosmic string loop collisions. The process of unzipping and string disentanglement has important effects on the evolution of networks containing different types of strings. Initially, one may fear that the overabundance of junctions and the string bound states may lead to a frustrated network of cosmic strings, preventing the network to reach a scaling regime. In an interesting simulation run by Urrestilla and Vilenkin [18] it was shown that the presence of junctions and bound states is not catastrophic. Indeed, it was shown that for a network containing two different types of strings, the contribution of the bound states to the population and length is negligible compared to that of the original strings. There may be two reasons for why the contribution of the junctions and bound states to the network's string length and number density is subdominant. First, cosmic strings move with very high velocities and can simply pass through each other, and no junctions form in the first place $[20-22,26]$. Second, and more curiously, junctions may materialize occasionally but they soon become unstable to unzipping. This was the subject of our current study.

To simplify the analysis, here we considered the simple case when the colliding loops have equal tensions and radii. In principle one can consider more general cases when loops have different tensions and configurations.

In examples of straight strings in collision [20-22], the junctions do not stop growing in time once they are formed. In contrast, we have demonstrated that for colliding loops unzipping phenomena take place. It is energetically costly for junctions to grow indefinitely. The junctions holding the external loops and those holding the internal loops behave differently. The junctions holding the external large loops start to unzip sooner than the junctions holding the internal small ones. The onset of unzipping and eventual loops disentanglement is determined by the parameters of the colliding loops such as their tensions, the angle of collision and their velocity.

\section{ACKNOWLEDGMENTS}

We would like to thank T. Kibble, R. Myers, S. SheikhJabbari, T. Vachaspati and A. Vilenkin for useful discussions and comments. H.F., J. K. and R. B. would like to acknowledge the hospitality of the KITPC, where this work was started, and the Perimeter Institute, where this work was finished. At McGill, this research has been supported by NSERC under the Discovery Grant program. R. B. is also supported by the Canada Research Chairs program.
[1] S. Sarangi and S. H. H. Tye, Phys. Lett. B 536, 185 (2002).

[2] M. Majumdar and A. Christine-Davis, J. High Energy Phys. 03 (2002) 056.

[3] S. H. Henry Tye, Lect. Notes Phys. 737, 949 (2008).

[4] T. W. B. Kibble, arXiv:astro-ph/0410073.

[5] A. C. Davis and T. W. B. Kibble, Contemp. Phys. 46, 313 (2005).
[6] M. Sakellariadou, Phil. Trans. R. Soc. A 366, 2881 (2008).

[7] B. Shlaer and M. Wyman, Phys. Rev. D 72, 123504 (2005).

[8] R. Brandenberger, H. Firouzjahi, and J. Karouby, Phys. Rev. D 77, 083502 (2008); T. Suyama, Phys. Rev. D 78, 043532 (2008).

[9] R. Brandenberger, H. Firouzjahi, J. Karouby, and S. 
Khosravi, J. Cosmol. Astropart. Phys. 01 (2009) 008; M. G. Jackson and X. Siemens, J. High Energy Phys. 06 (2009) 089; P. Binetruy, A. Bohe, T. Hertog, and D. A. Steer, arXiv:0907.4522.

[10] L. Leblond, B. Shlaer, and X. Siemens, Phys. Rev. D 79, 123519 (2009).

[11] E. J. Copeland, R. C. Myers, and J. Polchinski, J. High Energy Phys. 06 (2004) 013; G. Dvali and A. Vilenkin, J. Cosmol. Astropart. Phys. 03 (2004) 010; L. Leblond and S.-H.H. Tye, J. High Energy Phys. 03 (2004) 055; N. Barnaby, A. Berndsen, J. M. Cline, and H. Stoica, J. High Energy Phys. 06 (2005) 075.

[12] H. Firouzjahi, L. Leblond, and S. H. Henry Tye, J. High Energy Phys. 05 (2006) 047; H. Firouzjahi, J. High Energy Phys. 12 (2006) 031; K. Dasgupta, H. Firouzjahi, and R. Gwyn, J. High Energy Phys. 04 (2007) 093; M. Lake, S. Thomas, and J. Ward, arXiv:0906.3695.

[13] M. G. Jackson, N. T. Jones, and J. Polchinski, J. High Energy Phys. 10 (2005) 013; A. Hanany and K. Hashimoto, J. High Energy Phys. 06 (2005) 021; K. Hashimoto and D. Tong, J. Cosmol. Astropart. Phys. 09 (2005) 004; M. Eto, K. Hashimoto, G. Marmorini, M. Nitta, K. Ohashi, and W. Vinci, Phys. Rev. Lett. 98, 091602 (2007); M. G. Jackson, J. High Energy Phys. 09 (2007) 035.

[14] H. Firouzjahi, Phys. Rev. D 77, 023532 (2008).

[15] Y. Cui, S. P. Martin, D. E. Morrissey, and J. D. Wells, Phys. Rev. D 77, 043528 (2008).
[16] A. C. Davis, W. Nelson, S. Rajamanoharan, and M. Sakellariadou, J. Cosmol. Astropart. Phys. 11 (2008) 022.

[17] S. H. Tye, I. Wasserman, and M. Wyman, Phys. Rev. D 71, 103508 (2005); 71, 129906(E) (2005); L. Leblond and M. Wyman, Phys. Rev. D 75, 123522 (2007); A. Avgoustidis and E. P. S. Shellard, Phys. Rev. D 78, 103510 (2008); A. Rajantie, M. Sakellariadou, and H. Stoica, J. Cosmol. Astropart. Phys. 11 (2007) 021; M. Sakellariadou and H. Stoica, J. Cosmol. Astropart. Phys. 08 (2008) 038.

[18] J. Urrestilla and A. Vilenkin, J. High Energy Phys. 02 (2008) 037.

[19] L. M. A. Bettencourt and T. W. B. Kibble, Phys. Lett. B 332, 297 (1994).

[20] E. J. Copeland, T. W. B. Kibble, and D. A. Steer, Phys. Rev. Lett. 97, 021602 (2006).

[21] E. J. Copeland, T. W. B. Kibble, and D. A. Steer, Phys. Rev. D 75, 065024 (2007).

[22] E. J. Copeland, H. Firouzjahi, T. W. B. Kibble, and D. A. Steer, Phys. Rev. D 77, 063521 (2008).

[23] P. Salmi, A. Achucarro, E. J. Copeland, T. W. B. Kibble, R. de Putter, and D. A. Steer, Phys. Rev. D 77, 041701 (2008).

[24] N. Bevis and P. M. Saffin, Phys. Rev. D 78, 023503 (2008).

[25] N. Bevis, E. J. Copeland, P. Y. Martin, G. Niz, A. Pourtsidou, P. M. Saffin, and D. A. Steer, arXiv:0904.2127.

[26] A. Achucarro and R. de Putter, Phys. Rev. D 74, 121701 (2006). 